\title{
P45 Comparison of Systemic and Regional Vascular Parameters Using New Ultrasound Techniques: Are they Clinically Useful?
}

\author{
Pedro Forcada ${ }^{1,2, *}$, Miguel Sangiovanni ${ }^{1}$, Wanda Pampinella ${ }^{1}$, Oscar Montaña \\ ${ }^{1}$ dim Prevencion Cardiovascular - Buenos Aires - Argentina \\ ${ }^{2}$ Cardioarenales - Buenos Aires - Argentina
}

\section{ABSTRACT}

Background: US is a simple, reproducible and economic technique for vascular evaluation. New technologies using radiofrequency and multidimensional Doppler plus computer development allow to evaluate parameters like shear stress, Flow profile and vascular remodeling no invasively.

Objective: To compare the "regional" carotid evaluation with the accepted parameters of vascular disease like remodeling, arterial stiffness and endothelial function in hypertensive patients.

Methods: We evaluated 43 consecutive patients (18 healthy referent, 12 controlled HTN y 6 non controlled HTN) derived for NIVE (IMT, Plaques, PWV c-f and Endothelial Function) ("systemic parameters") and using a new US machine Resona $7^{\circ}$ with radiofrequency and multidimensional Doppler (VFlow) we obtained "regional parameters" of the common carotid artery like remodeling, arterial stiffness, wall shear stress and hemodynamic data like instantaneous speed and flow and speed gradient. The protocol was approved by LEC.

Results: All the systemic parameters (IMT, \%Plaques, PWV c-f and Endothelial Function) were significantly abnormal $(p<0.05)$ independently of BP control. The regional parameters and the regional hemodynamics showed an abnormal tendency without statistical significance. Correlation, regression and concordance tests showed that these parameters are concordant but not interchangeable.

Conclusion: The systemic and regional parameters where altered in hypertensive patients indicating the presence of associated vascular disease, independently of BP control. However, they were not interchangeable. These findings are limited due to the sample size and heterogeneity to yield a clinical application. Maybe, this pattern of regional vascular abnormalities, clearly different in hypertensives, could open a new field of investigation of vascular disease in hypertension.

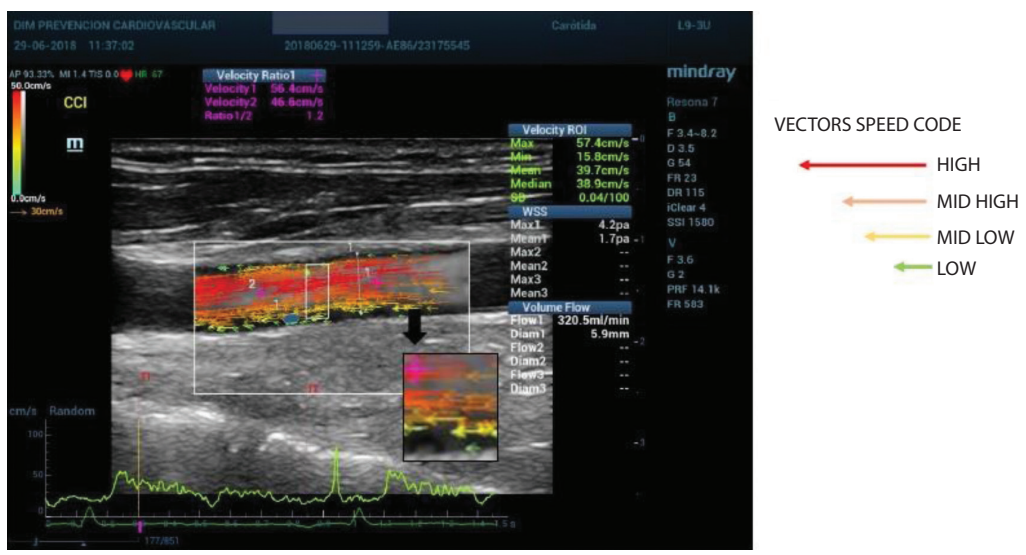

\section{REFERENCES}

[1] Nichols WW, O'Rourke MF, Vlachopoulos C. McDonald's blood flow in arteries. In: Arnold H, editor. Theoretical and clinical principles. 6th ed. London, England: CRC Press; 2011

[2] Goddi A, Bortolotto C, Fiorina I, Raciti MV, Fanizza M, Turpini E, et al. High-frame rate vector flow imaging of the carotid bifurcation. Insights Imaging 2017;8:319-28.

(c) 2019 Association for Research into Arterial Structure and Physiology. Publishing services by Atlantis Press International B.V. This is an open access article distributed under the CC BY-NC 4.0 license (http://creativecommons.org/licenses/by-nc/4.0/) 